\title{
Multiple Endocrine Neoplasia Type 1
}

\author{
GERARD M. DOHERTY, MD* \\ NW Thompson Professor of Surgery, University of Michigan, Ann Arbor, Michigan
}

\begin{abstract}
Multiple endocrine neoplasia-1 (MEN-1) is an autosomal dominant inherited syndrome that occurs due to inactivating mutations of the MEN1 gene locus, coding for a tumor-suppressor protein, menin. The components of MEN-1 are hyperparathyroidism due to multiple parathyroid adenomas, pancreatic neuroendocrine tumors, and pituitary adenomas, in addition to some less common neoplastic manifestations. Care of people with MEN-1 requires knowledge of the problems that may arise, and the best approaches to detect and care for the manifestations of this incurable, but manageable, disease.
\end{abstract}

J. Surg. Oncol. 2005;89:143-150. (c) 2005 Wiley-Liss, Inc.

KEY WoRDs: MEN-1; MEN1 gene; parathyroid disease; pancreas; pituitary

Multiple endocrine neoplasia type 1 is an autosomal dominant inherited syndrome that occurs due to inactivating mutations of the MENI gene locus, coding for a tumor-suppressor protein, menin. Inactivation of one copy of the menin protein-coding alleles, allows neoplasia to occur after an inactivating somatic mutation of the remaining allele (consistent with the Knudsen two-hit hypothesis). The clinical syndrome of MEN-1 classically includes hyperparathyroidism due to multiple parathyroid adenomas, pancreatic neuroendocrine tumors, and pituitary adenomas. In addition, MEN-1 carriers can have multiple lipomas, adrenal or thyroid adenomas, cutaneous angiofibromas, and bronchial or thymic carcinoid tumors.

\section{CLINICAL PRESENTATION}

The peak incidence of symptoms in women with MEN-1 is during the third decade of life, whereas the peak incidence in men is during the fourth decade [1]. MEN-1 can usually be detected in individuals from known MEN-1 kindreds by the age of 25 by clinical screening; if the gene defect in the affected family has been defined, direct genetic testing can be used at any age [2]. Specimens for sequencing of the MEN1 gene should only be processed if the clinical diagnosis in the proband is clear. Testing is not indicated for patients with an equivocal clinical diagnosis of MEN-1. More than onehalf of patients with MEN-1 have clinical involvement of more than one organ system, and approximately $20 \%$ have three affected endocrine glands. The frequency of clinical signs and symptoms, in descending order, is hypercalcemia, nephrolithiasis, peptic ulcer disease, hypoglycemia, headache, visual field loss, hypopituitarism, acromegaly, galactorrhea-amenorrhea, and rarely Cushing's syndrome. Patients with MEN-1 have a decreased life expectancy, with a $50 \%$ probability of death by age 50. One-half of the deaths are due to a malignant tumoral process or a sequela of the disease [3-5]. Separation of the patient's potential or actual problems into the various affected organs aids in the deliberate management of these complex patients.

\section{PARATHYROID DISEASE}

Asymmetric multigland primary hyperparathyroidism is the most frequent feature of MEN-1. Studies have demonstrated that there is a monoclonal abnormality in the enlarged parathyroid glands of patients with MEN-1, suggesting that the process in these glands may not be dependent on a circulating factor (hyperplasia) but rather occurs through inactivation of the MEN-1 gene in a precursor cell (neoplasia) [6]. The relationship of asymmetry of affected glands to patient age (younger patients more frequent asymmetric involvement, older patients with involvement of all glands) and the tumor suppressor genetic basis of the syndrome, favor the occurrence of multiple adenomas (neoplasia) rather than hyperplasia [7].

*Correspondence to: Gerard M. Doherty, MD, NW Thompson Professor of Surgery, University of Michigan, 2920 Taubman Center, 1500 East Medical Center Drive, Ann Arbor, MI 48109. Fax: 734-936-5830.

E-mail: gerardd@umich.edu

DOI 10.1002/jso.20181

Published online in Wiley InterScience (www.interscience.wiley.com). 
Primary hyperparathyroidism occurs in $88 \%-97 \%$ of MEN-1-affected patients. The diagnosis is dependent on the detection of elevated serum levels of calcium and parathyroid hormone. Primary hyperparathyroidism is usually the initially recognized clinical manifestation of patients with MEN-1, although in prospectively screened patients, other manifestations may be detected earlier biochemically $[2,8]$. The most frequent symptomatic tumor in teenagers with MEN-1 is insulinoma; occasional patients have clinical manifestations of ZollingerEllison syndrome (ZES) before primary hyperparathyroidism. The pathology associated with primary hyperparathyroidism is always multiple gland disease. Although some glands may appear grossly normal, the likelihood of finding normal-weight glands decreases as the age of the patient increases, consistent with the development of multiple parathyroid adenomas [7].

The surgical management requires a strategy that acknowledges that all of the parathyroid glands are, or will be, abnormal, and that the patient is better served by having a smaller amount of abnormal parathyroid tissue, than by having none at all. Options include removal of three and a half glands leaving a part of one gland in the neck, or removing all four parathyroid glands with immediate autograft of some of the parathyroid tissue into the musculature of the non-dominant forearm. Neither strategy yields ideal results. The incidence of recurrent or persistent hyperparathyroidism is $16 \%-$ $54 \%$, and the incidence of hypoparathyroidism is between $10 \%$ and $25 \%[9,10]$. Re-operations are frequently necessary, and similar principles should be applied.

\section{PANCREATIC ENDOCRINE DISEASE}

Malignant pancreatic endocrine tumors are the most common MEN-1-related cause of death in MEN-1 kindreds [3-5]. Pathologic examination of the duodenum and pancreas in patients with MEN-1 demonstrates multiple neuroendocrine tumors $[11,12]$. Tumors producing pancreatic polypeptide are the most common pancreatic endocrine tumor in MEN-1 patients, occurring in $80 \%-100 \%$. These tumors cause symptoms only due to the tumor mass itself and thus often present when tumor growth is advanced. Many patients develop functional pancreatic endocrine tumors, sometimes coincident with pancreatic polypeptide-producing tumors, of whom most have gastrinoma, approximately $20 \%$ insulinoma, $3 \%$ glucagonoma, and $1 \%$ vasoactive intestinal peptide (VIPoma).

The natural history of pancreatic disease in MEN-1 has been difficult to define in the past because of the rarity of the disease and significant variability in the virulence of the pancreatic malignancy. However, three recent studies have identified significant mortality associated with the pancreatic islet cell tumors, a natural history that demands intervention in that group of patients with virulent malignancy. The studies evaluated patients from Tasmania, and those followed at Washington University and the Mayo Clinic [3-5].

In the Washington University study, the database contained 34 distinct kindreds with 1,838 members. Reliable death data were available for 103 people and survival curves of MEN-1 patients who died from causes related to MEN-1, were compared to MEN-1 carriers who died from a non-endocrine cause, and to unaffected kindred member [3]. The ages of death were contrasted between affected and unaffected members of MEN-1 kindreds. Of $59 \mathrm{MEN}-1$ affected patients, 27 died directly of MEN-1-specific illness, and 32 died of non-MEN-1 causes. The MEN-1-specific deaths occurred younger (median 47 years) than either MEN-1 patients whose death was from some non-endocrine cause (median 60 years, $P<0.02$ ), or than all kindred members who did not die of MEN-1 disease (median 55 years, $P<0.05$ ). The causes of death in the MEN-1 patients included islet cell tumor (12 patients), ulcer disease (6 patients), hypercalcemia/uremia (3 patients), carcinoid tumor (6 patients), and non-endocrine malignancies (9 patients). MEN-1 carriers did not have a difference in survival compared to unaffected kindred members. Thus, of the Washington University MEN-1 patients, $46 \%$ died from causes related to their endocrine tumors after a median of 47 years, which was younger than family members who did not die of these tumors, and the most frequent cause of death, especially in the more recent years of the study, was pancreatic islet cell tumors. In addition, the metastatic islet cell tumors accounted for some of the youngest deaths in the study median age 46 (range 27-89).

The Tasman and Mayo Clinic series are similar. In the Tasman series, there were 46 deaths in patients with MEN-1, 20 of which were caused by components of the disease (44\%) [4]. Of these, only three were due to metastatic islet cell tumor; however, each of these three were in the most recent decade of the study, when the patients were best evaluated and characterized. The Mayo Clinic series included 60 deaths, of which only 17 were clearly related to MEN-1 (28\%), however, 10 of these were due to metastatic islet cell tumor [5]. The combined series includes 64 deaths clearly related to MEN-1, 43 of which were due directly to malignant neoplasms, and 25 of which were malignant islet cell tumors. Thus, the natural history of MEN-1 justifies an aggressive screening program with early therapeutic intervention when a tumor is identified.

To date, no studies have demonstrated that surgical resection of pancreatic neuroendocrine tumors in MEN-1 is beneficial. One study suggests that surgical resection 
of primary gastrinomas in patients with and without MEN-1 decreases the probability of the development of liver metastases, which is the most important negative predictor of survival [13]. Based on these data, patients with positive imaging studies and no evidence of unresectable metastases should undergo surgical exploration with intraoperative ultrasound. Tumors larger than $1 \mathrm{~cm}$ identified in the pancreatic head are enucleated, the duodenum is carefully explored by duodenotomy, and solitary or multiple tumors identified are resected; large tumors in the pancreatic body or tail are removed by distal pancreatectomy and splenectomy [14]. Resection of liver metastases from patients with MEN-1 may also be beneficial. Using this approach, while cure of ZES is unusual, resection should reduce the risk of subsequent metastatic disease. No data conclusively demonstrate that this approach increases survival, although case series are suggestive [15].

The surgical management of insulinoma and VIPoma in MEN-1 patients has more frequent biochemical cures than resection for gastrinoma. Medical management of the watery diarrhea in VIPoma is effective using either short-acting or depot somatostatin analogues. Hypoglycemia management in insulinoma is not reliable. Diazoxide and octreotide are available and may be useful for short-term treatment. In patients with MEN-1, insulinoma and VIPoma are frequently solitary large tumors. Resection may result in cure [15].

The most controversial issue in MEN-1 management is probably the decision to intervene in the pancreatic disease. Based on the data that implicate the pancreatic tumors as a potentially lethal lesion, and resection as the only option to prevent or delay this, all macroscopic tumors of the MEN-1 pancreas should be regarded as potentially malignant, and neither tumor size nor radiological findings or peptide production can safely be used as markers of malignancy [16]. Time may be an important risk factor for mutagenetic events essential for malignant transformation [17]. In addition, 50\% of middle-aged patients display metastases. The prevention of death from metastatic islet cell tumor requires pancreatic surgery before frank clinical syndromes occur, in relatively young MEN-1 patients, based on active surveillance programs.

Surveillance for pancreatic endocrine tumors in patients with the MEN-1 mutation is a critical part of the management of adult patients. The options for surveillance include (1) hormone measurements, (2) crosssectional imaging, (3) somatostatin-receptor scintigraphy, and (4) endoscopic ultrasonography. Although pancreatic endocrine tumors can occur in people with an MEN-1 mutation during childhood, they are almost always insulinomas that declare themselves by symptoms. Surveillance for these tumors is not necessary until adulthood.

\section{Biochemical Surveillance}

Hormone measurements have been useful to screen for pancreatic tumors in MEN-1 because of the frequent functional nature of the tumors [2,18,19]. Efficient programs have been designed for clinical recognition of the lesions. Patients can be screened biochemically, either with or without a provocative agent, such as a standard meal, calcium or secretin. The results of the biochemical testing can then be used to select patients for imaging. Biochemical testing is useful for annual or semiannual evaluation of asymptomatic gene carriers. Patients with specific symptoms require investigation by other means.

The advantage of biochemical testing is that patients can be identified as harboring functional pancreatic endocrine tumors in a pre-symptomatic state. The disadvantages of the biochemical testing are that some tumors make insufficient hormone to be identified, and some patients harbor tumors that are identifiable biochemically, but not imageable at that point [2]. This can lead to extensive testing and possibly operation that is unlikely to benefit the patient clinically.

A standard annual biochemical screening program has been advocated by Dr. Skogseid and her colleagues from the University of Uppsala. In their program, within their annual visit that also includes evaluation for parathyroid and pituitary disease, patients have baseline levels of insulin, proinsulin, glucagon, pancreatic polypeptide, and gastrin, followed by meal-stimulated levels of gastrin and pancreatic polypeptide. This approach identified pancreatic tumors as the initial presenting abnormality in several patients, and identified tumors at an early stage. However, the biochemical screening, and particularly the meal test, can generate some false-positive results. Mild elevations in the biochemical screening must be repeated after an interval to establish confidence that it reflects pancreatic endocrine neoplasia [20].

Efforts to replace the standard meal test with a provocative test using calcium and secretin have been stalled by the unavailability of secretin, and the uncomfortable nature of the calcium infusion [21]. However, the infusion of calcium $(2 \mathrm{mg} / \mathrm{kg})$ and secretin $(2 \mathrm{U} / \mathrm{kg})$ in ten known MEN-1 carriers did demonstrate marked differences in induced (2-min peak) serum levels of pancreatic polypeptide, gastrin, and insulin compared to normal volunteers. The elevation of hormone levels was also correlated with the presence of imageable tumors.

\section{Cross-Sectional Imaging and Somatostatin-Receptor Scintigraphy Surveillance}

Evaluation with cross-sectional imaging, such as computed tomography scan, can demonstrate tumors in the pancreas, regional lymph nodes, and hepatic metastases (Figs. 1 and 2). This modality has the advantage of 

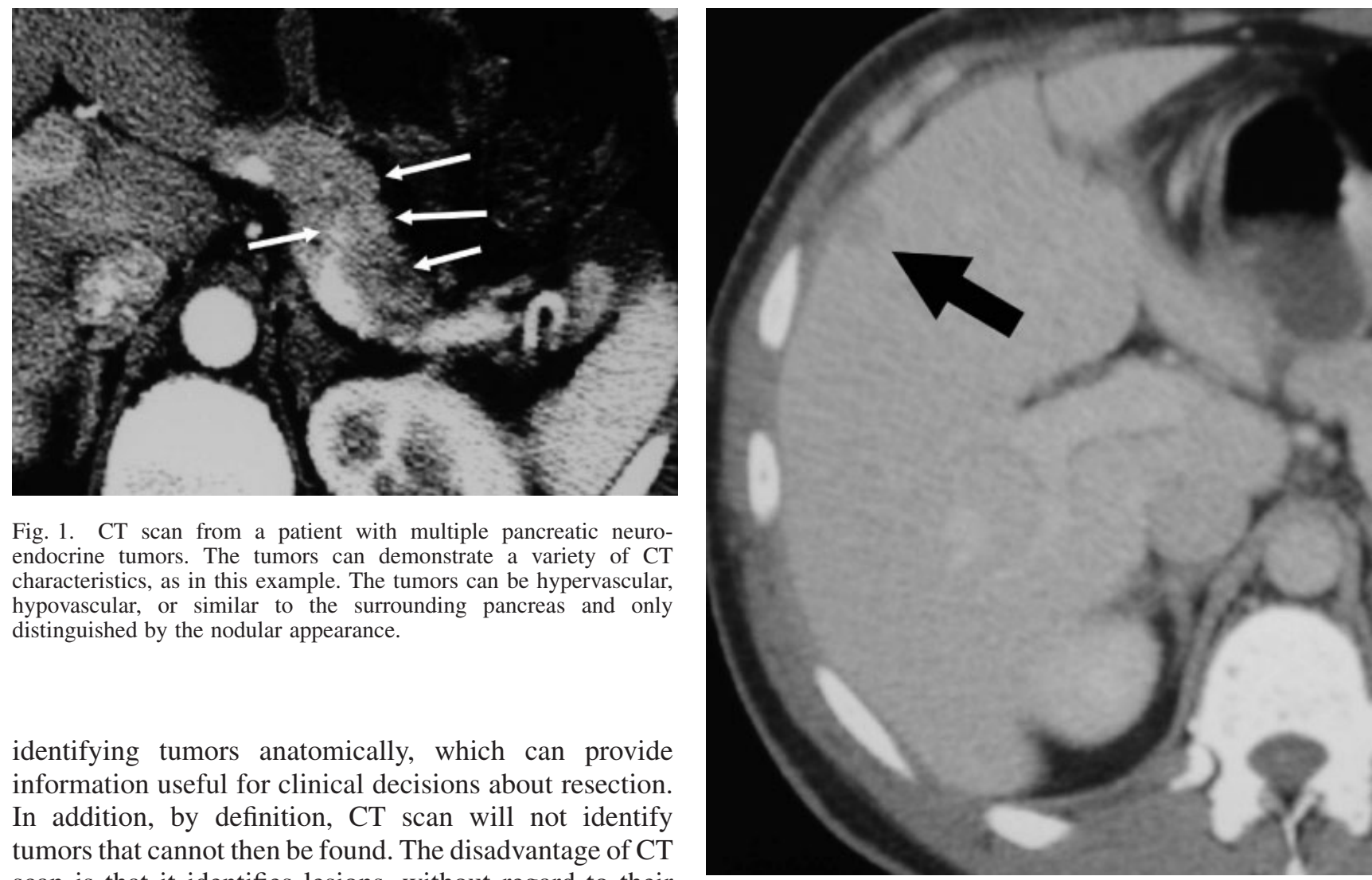

Fig. 1. CT scan from a patient with multiple pancreatic neuroendocrine tumors. The tumors can demonstrate a variety of CT characteristics, as in this example. The tumors can be hypervascular, hypovascular, or similar to the surrounding pancreas and only distinguished by the nodular appearance.

identifying tumors anatomically, which can provide information useful for clinical decisions about resection. In addition, by definition, CT scan will not identify tumors that cannot then be found. The disadvantage of CT scan is that it identifies lesions, without regard to their histology or function. Thus, lesions identified in the pancreas, or more commonly, in adjacent nodes, may be irrelevant findings independent of the MEN-1 syndrome. Prospective studies that have evaluated the sensitivity of CT imaging to detect pancreatic endocrine tumors, have found that it is significantly less sensitive than biochemical testing in identifying early disease [2]. Thus, for surveillance, CT is less useful, but for establishing the anatomic extent of disease after biochemical diagnosis, CT is critical.

Somatostatin-receptor scintigraphy can identify lesions in and around the pancreas and liver, as well as throughout the remainder of the body (Fig. 3). SRS has the advantage of identifying tumors functionally, based on the size of the tumor mass and the concentration of somatostatin receptors [22,23]. The disadvantage of SRS lies in its lack of anatomic specificity. The nuclear medicine images, even with SPECT cross-sectional imaging, provide only general anatomic definition with respect to surrounding organs. SRS must always, therefore, be combined with CT scan or other anatomic imaging techniques to provide anatomic correlates to the scintigraphic findings. In addition, SRS has the advantage of identifying other potential sites of disease, including intrathoracic disease from either metastases of the pancreatic primary, or carcinoid tumors of the thymus or bronchus.

Fig. 2. The typical appearance of a metastatic lesion to the liver from a pancreatic neuroendocrine tumor (same patient as in Fig. 1). This lesion, and two other metastases, and the patient multiple pancreatic primary tumors, were all resected, with resolution of his clinical insulinoma syndrome.

In a prospective study of SRS in MEN-1 patients, 37 SRS studies were performed in $29 \mathrm{MEN}-1$ patients [22]. SRS identified occult tumor in 36\% (4/11) of patients with only biochemical evidence of neuroendocrine tumor; two patients went on to resection. SRS showed tumor in 79\% (15/19) of patients with CT-demonstrated tumor; $30 \%$ (6/20) of the SRS lesions were occult on CT. Conversely, 55\% (16/29) of CT-identified lesions were occult on SRS. SRS found distant disease in 21\% (6/29) of patients. In previously operated patients, SRS found tumor in $40 \%$ (4/10) of patients, again with both new positives and false negatives compared to other imaging. SRS also had three important false-positive results, including one patient who had laparotomy with no tumor identified. Thus, SRS was useful in identifying otherwise occult neuroendocrine tumors in MEN-1 patients and substantially altered management. However, SRS also has significant false-positive and false-negative results which demand correlation with other studies. 


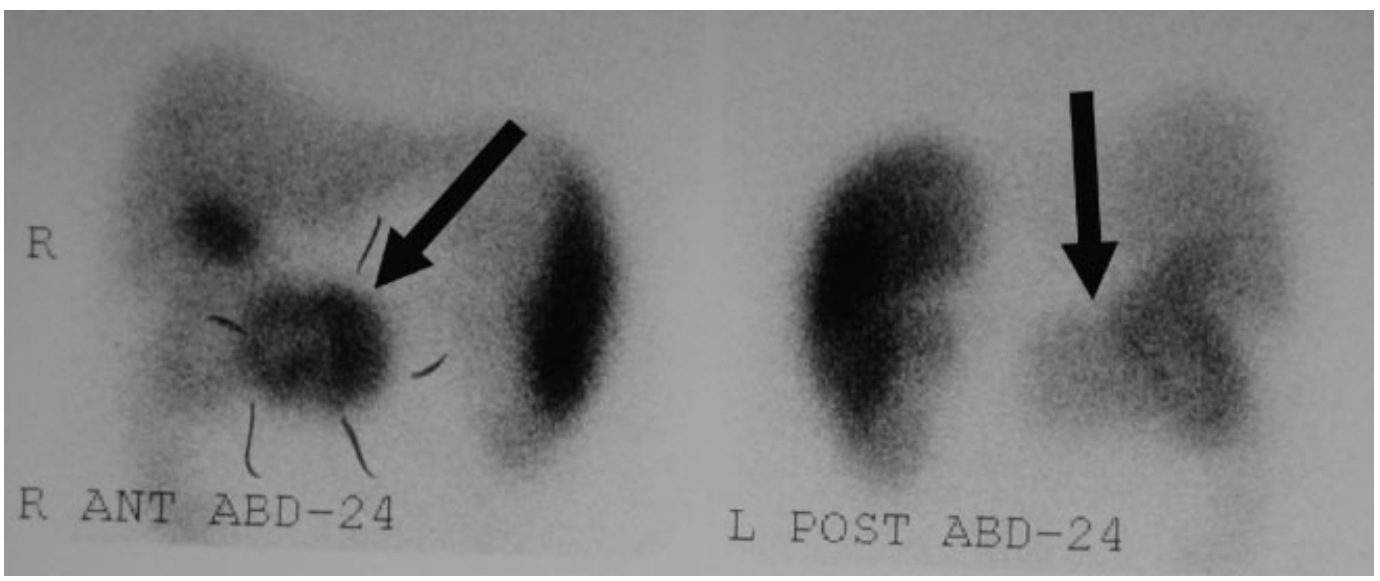

Fig. 3. Somatostatin-receptor scintigraphy for the detection of pancreatic neuroendocrine tumor. The image is very helpful, but only if carefully correlated with cross-sectional imaging to further localize the lesions.

\section{Endoscopic Ultrasound Surveillance}

Endoscopic ultrasound is the most sensitive imaging modality for small pancreatic endocrine tumors. Its advantage lies in the ability to image the parenchyma of the pancreas and the immediately peri-pancreatic lymph nodes with exquisite precision. The disadvantage of the EUS is that it cannot image any of the other potential sites of disease, such as the liver or chest. In a retrospective review of EUS in MEN-1 patients screened at the University of Michigan, 14 of 15 asymptomatic patients were found to have pancreatic tumors (12 with multiple lesions) with a median size of $1.5 \mathrm{~cm}$ (range $0.6-6.5 \mathrm{~cm}$ ) [24]. Many of these lesions were at or below the level of reliable detection of other imaging modalities, such as CT scan, and the EUS also provided detailed anatomic information useful for operative planning. The EUS was not useful for identifying duodenal wall gastrinomas, which are frequent in MEN-1. Because of its tremendous sensitivity in the pancreas, but limited anatomic scope, the EUS may be useful for a specific role in MEN-1 screening, to identify otherwise occult tumors in the asymptomatic patient.

A unified surveillance plan for pancreatic endocrine tumors in MEN-1 should take advantage of the strengths of each of these modalities. Biochemical assessment of hormones can lead to detailed investigation, but should probably not be the sole method. As the most sensitive test, biochemical evaluation can reassure clinicians and patients, and allow for other examinations at greater intervals if the biochemistry remains normal from year to year. For imaging examinations, a combination of CT scan and SRS provides the best coverage of the abdomen and thorax, to identify anatomic correlates to the functional lesions revealed by biochemical testing, and should be done at some interval even in the presence of normal biochemistry, to evaluate for non-functional lesions. Finally, the EUS appears to be useful in providing a very sensitive assessment of the pancreas, and may be especially useful in patients with biochemical evidence of tumor, but no imageable disease on SRS or CT scan. This may also be of particular value in patients who have had a previous partial pancreatectomy, which can complicate the interpretation of the CT scan.

\section{Management of the Functional Hormonal Syndrome}

The hormonal syndromes associated with functional PETs in MEN-1 should be managed as best as possible prior to operation. The management of ZES is very nearly perfect with proper medical regimens. Acid-suppression therapy resolves the peptic ulcer disease and diarrhea. Insulinoma syndrome is not well-palliated medically, and requires operative resection in order to relieve the syndrome. Management of VIPoma and glucagonoma syndromes are greatly facilitated by the use of a somatostatin analogue (octreotide) which can be administered monthly as a long-acting formulation.

\section{Operative Management of MEN-1 Pancreatic Disease}

The operative management must be individualized for each patient based on their pattern of disease. The principles are complete tumor resection and preservation of pancreatic function, by preserving as much grossly normal pancreas as possible, while minimizing the morbidity of the procedure [25-27]. In practice, this often results in the subtotal resection of the distal pancreas, and enucleation of tumors in the head of the pancreas and duodenum. This operation removes the gross disease in the distal pancreas, while preserving most of the pancreatic mass in the head, and avoiding the need for a pancreatic anastamosis. For patients with 
bulky disease in the head of the pancreas, however, which may not be amenable to enucleation, a better option may be pancreaticoduodenectomy, and enucleation of any tumors in the tail. In all patients with gastrinoma, the duodenum should be opened and submucosal tumors resected. Occasional patients may have disease that can be completely removed without formal pancreatic resection. Total pancreatectomy is rarely indicated, or necessary, to meet the goal of complete tumor resection.

A contemporary series of pancreatectomy in MEN-1 patients catalogues the operations and their outcomes at Washington University between 1993 and 1999 [26]. Twenty-one consecutive patients with tumors identified through an integrated disease screening program underwent exploration, with the twin goals of complete tumor resection and preservation of grossly normal pancreas. In this series of patients, 5 required pancreaticoduodenectomy, 11 had non-whipple pancreatic resections, and 5 underwent enucleation of limited neuroendocrine tumors. The morbidity of these operations was significant, and two patients died in the perioperative period. One patient developed fatal viral encephalitis 6 weeks after an otherwise uneventful recovery. The other mortality was in an elderly man with prohibitive pulmonary function $\left(\mathrm{FEV}_{1}<700 \mathrm{ml}\right)$ and uncontrollable hypoglycemia from an insulinoma, who died of pulmonary failure following resection. The morbidity of operation included a $14 \%$ rate of anastomotic leak and abscess and a $10 \%$ rate of wound infection. In these patients, the operative strategy was able to render them disease-free in the short term, but follow-up is currently insufficient to demonstrate efficacy in this series.

In a similar study with more extended follow-up, the University of Uppsala group detailed their approach in 20 patients, 12 of whom were symptomatic and 8 patients identified by screening [17]. They followed the same guidelines of complete tumor resection with preservation of functional pancreatic mass. To date, none of the patients whose tumors were identified by screening in their study have developed metastatic disease. While further follow-up of both this cohort and the Washington University series is necessary to determine whether this strategy will have a long-term benefit in reducing the occurrence of death from malignant islet cell tumors, this currently appears to be the most viable strategy.

One study has sufficient follow-up to allow some conclusions regarding the effects of operation on outcome [28]. Between 1967 and 2003, 39 patients, ages 19-58 (mean age 37.1), had abdominal operations for their PETs: 26 with ZES, 4 with hypoglycemia, 3 with both ZES and hypoglycemia, and 6 with non-functional tumors. Fifteen of these 39 patients had malignant disease on initial abdominal operation. Twenty-four of 39 patients have not required abdominal re-operation, 17 of whom have available follow-up data. Of these 17 patients, 11 have biochemical evidence of disease recurrence (elevated gastrin, insulin or pancreatic polypeptide), while 6 have no biochemical evidence of recurrence. A total of 30 abdominal re-operations were performed in 15 patients. Fourteen of 15 patients undergoing one or more re-operations developed evident malignant disease by their most recent operation. Nine of 13 reoperative patients with follow-up data have evidence of disease recurrence. Functional outcomes available in 20 patients showed that 10 patients require insulin injections and 6 require oral hypoglycemics. Ninety percent have no abdominal pain or nausea/vomiting, while four are unable to return to work secondary to this disease. Seven patients have died since initial operation, two from sequalae of their pancreaticoduodenal NETs. These data demonstrate that treatment of MEN-1 PETs is met with frequent recurrence (Table I), even after re-operation to resect additional or recurrent disease (Table II), and some treatment-related morbidity and mortality. Most patients (22 of 39) eventually demonstrated malignant growth, but with this strategy, few died of this disease. The overall survival of our series of MEN-1 patients undergoing abdominal operation for pancreaticoduodenal NETs shows that the median survival age for patients managed with this strategy has not been reached. Thus, this appears to be a viable approach to changing the natural history defined in other studies.

\section{PITUITARY DISEASE}

Pituitary tumors occur in $54 \%-80 \%$ of patients with MEN-1. Symptoms may be due to local encroachment

TABLE I. Incidence of MEN-1 Pancreaticoduodenal Disease Recurrence*

\begin{tabular}{lcccc}
\hline & Number of & & $\begin{array}{c}\text { Median total } \\
\text { follow-up time } \\
\text { (years) }\end{array}$ & $\begin{array}{c}\text { Range of } \\
\text { follow-up } \\
\text { (years) }\end{array}$ \\
\hline No recurrence & 8 & 20.5 & 4.8 & $0.8-12.3$ \\
Recurrence and reoperation & 15 & 38.5 & 9.9 & $3.1-30.0$ \\
Recurrence and no reoperation & 12 & 30.8 & 6.3 & $0.1-11.0$ \\
Lost to follow-up & 4 & 10.3 & 0.0 & \\
\hline
\end{tabular}

*From: Hausmann M et al. [28]. 
TABLE II. Incidence of Pancreaticoduodenal Disease Recurrence in the Reoperative Group*

\begin{tabular}{lcccc}
\hline Recurrence pattern & Number of & & $\begin{array}{c}\text { Median time interval } \\
\text { between reoperations } \\
\text { patients }\end{array}$ & $\begin{array}{c}\text { Median total follow-up time } \\
\text { since initial operation } \\
\text { (years) and range (years) }\end{array}$ \\
\hline No recurrence after first reoperation & 0 & 0.0 & 0 & 0 \\
Recurrence and additional reoperation performed & 10 & 66.7 & $3.1(0.1-27.8)$ & $13.3(3.1-30.0)$ \\
Recurrence and no additional reoperation performed & 4 & 26.7 & 0 & $8.1(4.3-10.9)$ \\
Lost to follow-up & 1 & 6.7 & 0 & 9.3 \\
\hline
\end{tabular}

*From: Hausmann M et al. [28].

including headache and visual field defects. In a review of series, the most common tumor was a prolactinoma $(41 \%-76 \%)$, followed by growth hormone-secreting tumor, nonfunctional tumor, and rarely adrenocorticotropic hormone-or thyroid-stimulating hormonesecreting tumors [29]. Men with prolactinoma may be unable to achieve a penile erection, whereas women may have galactorrhea and infertility. Growth hormonesecreting tumors $(25 \%)$ result in acromegaly. Cushing's syndrome can also result from release of adrenocorticotropic hormone-like material from a pancreatic islet cell tumor or a foregut carcinoid tumor (ectopic adrenocorticotropic hormone). Prolactinomas are generally treated by dopamine receptor agonists (bromocriptine, pergolide, cabergoline). Transsphenoidal pituitary surgery may be indicated to control any detectable pituitary mass lesion in patients with MEN-1. Incompletely resected patients may also be treated with bromocriptine.

\section{LESS TYPICAL FEATURES}

Adrenal abnormalities may occur in $27 \%-36 \%$ of patients with MEN-1. The most common abnormality is a benign, nonfunctional cortical adenoma, although adrenal cortical carcinomas and hyperplasia may also occur [30]. Adrenal cortical hyperfunction may rarely be found secondary to an adrenal tumor. Adrenal cortical neoplasms are usually nonfunctional in patients with MEN-1. Thyroid adenomas also occur in approximately 5\%-30\% of patients with MEN-1 and have little clinical significance. Lipomas are seen with greater frequency in patients with MEN-1, as are cutaneous angiofibromas and collagenomas [31].

Gastric carcinoid tumors develop in 7\%-30\% of patients with MEN-1 with ZES. They arise from gastric enterochromaffin-like cells and thus are also call ECLomas [32,33]. Approximately 18\% have metastases, the primary tumors are usually multiple, and they generally pursue an indolent course. They are rare in patients with MEN-1 without ZES.

Thymic carcinoids occur in $0 \%-8 \%$ of patients with MEN-1, are almost exclusively in men, are usually asymptomatic, and are not associated with Cushing's or carcinoid syndrome [34,35]. They pursue an aggressive course with distant metastases and are an increasing cause of death in older men with MEN-1. In contrast to parathyroid, pituitary and pancreatic tumors, thymic carcinoids do not show 11q13 LOH and therefore likely have a different pathogenesis.

Bronchial carcinoid tumors occur in $0 \%-8 \%$ of patients with MEN-1. Eighty percent are in women and $74 \%$ are benign; however, they are an occasional cause of death.

\section{FOLLOW-UP}

Continued surveillance for components of MEN-1 is necessary for all patients. This is a syndrome that one can manage, but not cure, and thus it requires continued attention. Patients should be assessed at least annually and more frequently if indicated by the tempo of their disease. Ongoing surveillance follows the same pattern and uses the same modalities as the screening in unoperated patients. The useful modalities include biochemical hormone screening for evidence of parathyroid, pituitary and pancreatic disease, and pancreatic imaging with CT scan, SRS, and possibly EUS. The usual interval between follow-up assessments for an asymptomatic patient is 1 year.

\section{REFERENCES}

1. Brandi ML, Gagel RF, Angeli A, et al.: Guidelines for diagnosis and therapy of MEN type 1 and type 2. J Clin Endocrinol Metab 2001;86:5658-5671.

2. Skogseid BS, Eriksson B, Lundqvist G, et al.: Multiple endocrine neoplasia type 1: A 10-year prospective screening study in four kindreds. J Clin Endocrinol Metab 1991;73:281-287.

3. Doherty GM, Olson JA, Frisella MM, et al.: Lethality of multiple endocrine neoplasia type I. World J Surg 1998;22:581-586; discussion 586-587.

4. Wilkinson S, Teh BT, Davey KR, et al.: Cause of death in multiple endocrine neoplasia type 1. Arch Surg 1993;128:683-690.

5. Dean PG, van Heerden JA, Farley DR, et al.: Are patients with multiple endocrine neoplasia type 1 prone to premature death? World J Surg 2000;24:1437-1441.

6. Thakker RV, Bouloux P, Wooding C, et al.: Association of parathyroid tumors in multiple endocrine neoplasia type 1 with loss of alleles on chromosome 11. N Engl J Med 1989;321:218 224.

7. Doherty GM, Lairmore T, Moley JF, et al.: MEN-1 parathyroid adenoma development over time. World J Surg 2004; Submitted. 
8. Skogseid B, Oberg K: Experience with multiple endocrine neoplasia type 1 screening. J Intern Med 1995;238:255-261.

9. Hellman P, Skogseid B, Oberg K, et al.: Primary and reoperative parathyroid operations in hyperparathyroidism of multiple endocrine neoplasia type 1. Surgery 1998;124:993-999.

10. Rizzoli R, Green J III, Marx SJ: Primary hyperparathyroidism in familial multiple endocrine neoplasia type I: Long-term follow-up of serum calcium levels after parathyroidectomy. Am J Med 1985;78:467-474.

11. Pipeleers-Marichal M, Donow C, Heitz PU, et al.: Pathologic aspects of gastrinomas in patients with Zollinger-Ellison syndrome with and without multiple endocrine neoplasia type I. World J Surg 1993;17:481-488.

12. Pipeleers-Marichal M, Somers G, Willems G, et al.: Gastrinomas in the duodenums of patients with multiple endocrine neoplasia type 1 and the Zollinger-Ellison syndrome [comment]. N Engl J Med 1990;322:723-727.

13. Fraker DL, Norton JA, Alexander HR, et al.: Surgery in Zollinger-Ellison syndrome alters the natural history of gastrinoma. Ann Surg 1994;220:320-330.

14. Lairmore TC, Chen VY, DeBenedetti MK, et al.: Duodenopancreatic resections in patients with multiple endocrine neoplasia type 1. Ann Surg 2000;231:909-918.

15. Norton JA, Alexander HR, Fraker DL, et al.: Comparison of surgical results in patients with advanced and limited disease with multiple endocrine neoplasia type 1 and Zollinger-Ellison syndrome. Ann Surg 2001;234:495-505; discussion 505-506.

16. Lowney JK, Frisella MM, Lairmore TC, et al.: Islet cell tumor metastasis in multiple endocrine neoplasia type I: Correlation with primary tumor size. Surgery 1998;124:1043-1049.

17. Skogseid B, Oberg K, Eriksson B, et al.: Surgery for asymptomatic pancreatic lesion in multiple endocrine neoplasia type 1 World J Surg 1996;20:872-877.

18. Skogseid BS, Rastad J, Oberg K: Multiple endocrine neoplasia type 1: Clinical features and screening. Endocrinol Metab Clin North Am 1994;23:1-18.

19. Vasen HF, Lamers CBH, Lips CJM: Screening for the multiple endocrine neoplasia syndrome type 1. Arch Int Med 1989;149: $2717-2722$.

20. Skogseid BS, Oberg K, Benson L, et al.: A standardized meal stimulation test of the endocrine pancreas for early detection of pancreatic endocrine tumors in multiple endocrine neoplasia type 1 syndrome: Five years experience. J Clin Endocrinol Metab 1987;64:1233-1240.

21. Olson JA, Debenedetti M, Wells SA, et al.: Islet cell neoplasia in multiple endocrine neoplasia, type 1: Development of a provocative screening test. Surgical Forum 1996;47:170-173.
22. Yim JH, Siegel BA, DeBenedetti MK, et al.: Prospective study of the utility of somatostatin receptor scintigraphy in the evaluation of patients with multiple endocrine neoplasia type I. Surgery 1998:124:1037-1042

23. Meko JB, Doherty GM, Siegel BA, et al.: Evaluation of somatostatin-receptor scintigraphy for detecting neuroendocrine tumors. Surgery 1996;120:975-984.

24. Gauger PG, Scheiman JM, Wamsteker E-J, et al.: An aggressive strategy of early anatomic screening and operative treatment of pancreatic endocrine tumors in asymptomatic patients with multiple endocrine neoplasia type 1. Br J Surg 2002; (in press).

25. Thompson NW. Surgical treatment of the endocrine pancreas and Zollinger-Ellison syndrome in the MEN I syndrome. Henry Ford Hosp Med J 1992;40:195-198.

26. Lairmore TC, Chen VY, DeBenedetti MK, et al.: Duodenopancreatic resections in patients with multiple endocrine neoplasia type 1 (MEN 1). Ann Surg 2000;231:909-918.

27. Skogseid B, Grama D, Rastad J, et al.: Operative tumour yields obviates preoperative pancreatic tumor localization in multiple endocrine neoplasia type 1. J Intern Med 1995;238:281 288.

28. Hausmann MS, Thompson NW, Gauger PG, et al.: The surgical management of MEN-1 pancreaticoduodenal neuroendocrine disease. Surgery 2004; (in press).

29. Metz DC, Jensen RT, Bale AE, et al.: Multiple endocrine neoplasia type 1: Clinical features and management. In Bilezekian JP, Levine MA, Marcus R, editors. The parathyroids, 0 edn New York: Raven Press Publishing; 1994. pp 591-646.

30. Skogseid B, Rastad J, Gobl A, et al.: Adrenal lesion in multiple endocrine neoplasia type 1. Surgery 1995;118:1077-1082.

31. Marx S, Spiegel AM, Skarulis MC, et al.: Multiple endocrine neoplasia type 1: Clinical and genetic topics. Ann Int Med 1998; 129:484-494.

32. Bashir S, Gibril F, Ojeaburu JV, et al.: Prospective study of the ability of histamine, serotonin or serum chromogranin A levels to identify gastric carcinoids in patients with gastrinomas. Aliment Pharmacol Ther 2002;16:1367-1382.

33. Bordi C, Corleto VD, Azzoni C, et al.: The antral mucosa as a new site for endocrine tumors in multiple endocrine neoplasia type 1 and Zollinger-Ellison syndromes. J Clin Endocrinol Metab 2001; $86: 2236-2242$.

34. Teh BT, Zedenius J, Kytola S, et al.: Thymic carcinoids in multiple endocrine neoplasia type 1. Ann Surg 1998;228:99105.

35. Gibril F, Chen YJ, Schrump DS, et al.: Prospective study of thymic carcinoids in patients with multiple endocrine neoplasia type 1. J Clin Endocrinol Metab 2003;88:1066-1081. 